\title{
VAGINAL BACTERIAL PROFILE IN PREGNANT WOMEN
}

\section{Anton G. Petrov, Milena D Karcheva ${ }^{1}$, Teodora V. Marinova- Balgaranova², Rositsa V. Petrova ${ }^{3}$, Verena K. Vasileva 4}

\author{
Department of Obstetrics and \\ Gynecology, \\ Medical University - Pleven \\ ${ }^{1}$ Sector of Epidemiology, Parasitology \\ and Tropical medicine, \\ Medical University - Pleven \\ ${ }^{2}$ Department of Microbiology, \\ Medical University - Pleven \\ ${ }^{3}$ Sector of Disaster Medicine, \\ Medical University - Pleven \\ ${ }^{4}$ Unit of Obstetrics and Gynecology, \\ MHAT Lukovit Ltd.
}

\author{
Corresponding Author: \\ Anton G. Petrov \\ Department of Obstetrics and Gynecology, \\ Medical University - Pleven \\ 1, Sv. Kliment Ohridski Str. \\ Pleven, 5800 \\ Bulgaria \\ e-mail: antongeorgiev@abv.bg
}

Received: February 02, 2021

Revision received: March 28, 2021

Accepted: May 27, 2021

\section{Summary}

This study aimed to determine the bacterial profile of vaginal samples from pregnant women and the prevalence of identified microorganisms. Materials and methods: A prospective epidemiological study was conducted on 150 pregnant women admitted to the Clinic of Obstetrics and Gynecology at Dr. G. Stranski University Hospital in Pleven. Vaginal secretion samples were collected from all the 150 women. Direct microscopy of native smears prepared by the wet mount method to detect Trichomonas vaginalis and direct microscopy of Gram-stained smears was performed. Aerobic culture studies were conducted to identify pathogenic bacteria and Sabouraud glucose agar (SGA) to detect the presence of Candida spp. Samples were also collected from 107 of the women for PCR tests for mycoplasma detection. Results: The highest relative share in the study was women aged 21 to 35 years $104(69.33 \%)$. Of all the hospitalized and examined pregnant women, 87 (57.99\%) had medical problems during the current pregnancy and postpartum complications, and 63 women (42\%) gave birth without accompanying complications. In $86(57.32 \%)$ of the women, bacteria were detected by direct microscopy of Gram-stained smears. Of these, $24(16 \%)$ had gram-negative rods, 28 (18.66\%) had gram-positive cocci. In 34 (22.66\%), we found gram-negative and gram-variable pleomorphic bacteria. The aerobic culture study revealed microbiological growth in 44 $(29.33 \%)$ of the women investigated. Conclusions: Studies on the profile of the vaginal microbiota in pregnant women and the spread of pathogenic microorganisms are essential for make prognoses for pregnancy outcome, evaluating the risk of developing a maternal-fetal infection, and discussing options for timely treatment.

Keywords: pregnancy, vaginal microbiota, microbiome, bacteria, infections

\section{Introduction}

The totality of all types of microorganisms that usually inhabit human skin and mucous membranes are united in the concept of microbiota [1]. Human microbiota consists of four populations: in the oral cavity, the gastrointestinal tract, on the skin, and in the vagina and urinary tract. It is a collection of all the genes microorganisms contained in the microbiota. The human microbiota can function as mutualistic (symbiotically helpful), commensal (neither harmful 
nor beneficial), or pathogenic factor (detrimental to the host). The resident flora that generally inhabits the vagina and the urinary tract protects against invading pathogenic microorganisms. The vaginal microflora contains 200 aerobic and anaerobic species [2]. The vagina is a source of amniotic infection in $95 \%$ of all cases. During pregnancy, the vaginal microbiota undergoes many changes under the influence of hormones, immunity, and other factors. The microbiome can change depending on the environment, weight, and diet, among other factors [3]. Of the ten main types in the vaginal ecosystem, Lactobacillus spp. constitute the majority $(95.9 \%)$ of the vaginal microbiota in healthy women [4].

Pregnancy leads to a change in the structure of the bacterial vaginal microbiome. The composition and organization of the vaginal microbiome during pregnancy may increase the incidence of adverse pregnancy outcomes [3]. The most common complications are premature birth, group A streptococcal infection (GAS), and infections of the newborn.

Microorganisms can be found in fetal membranes during any period of pregnancy, including those born at term by cesarean section. Infectious diseases account for up to $30 \%$ of neonatal deaths ( 1 million deaths per year) and $50 \%$ or more of all stillbirths in low- and middleincome countries. In contrast, in high-income countries, only $10-25 \%$ of stillbirths are caused by infection, according to Goldenberg et al. [5].

Escherichia coli spp. is the most common microorganism isolated in hospitalized pregnant women (43\%). Most women admitted to intensive care units with $\mathrm{E}$. coli infection have developed sepsis during pregnancy.

Another common bacterial species is Enterobacter spp. They act as opportunistic pathogens (E. cloacae, E. aerogenes, E. gergoviae, and E. agglomerans). Among newborns, Enterobacter spp. cause nosocomial infections (NI), and the high mortality suggests a high virulence of these microorganisms [6,7]. Enterobacter spp. are also associated with NI [8]. Enterococci are common etiological causes of nosocomial infections. According to some authors, they are the second most common cause of genitourinary tract infections in hospitals. E. faecalis is one of the most common pathogens found in patients with urinary tract infections
[9].

Klebsiella spp. are ubiquitous. In humans, they colonize the skin, pharynx, or gastrointestinal tract. The Klebsiella spp. are the most common bacterial pathogens in newborns in Israel, Ethiopia, India, and Mexico and account for mortality rates of about $66.6 \%$ [10]. Ballén V. et al. found that the prevalence of $\mathrm{K}$. pneumoniae in pregnant women was $0.6 \%$. There are reports of ascending infections caused by virulent strains of $\mathrm{K}$. pneumoniae, leading to acute placental infection, chorioamnionitis, and intrauterine fetal death [11,12].

Streptococcus spp. are a genus of grampositive cocci, many of which are facultative anaerobes. A group A streptococcal infection (GAS) can be life-threatening for women. The condition can occur during pregnancy or childbirth. GAS can also cause invasive infections such as endometritis, necrotizing fasciitis, or toxic shock syndrome, and puerperal sepsis caused by Group A streptococci remains a leading cause of maternal and infant mortality worldwide. These cocci can lead to sepsis and a very high mortality rate, ranging from 30 to $50 \%$.

Streptococcus agalactiae, belonging to group B streptococci (GBS), is among the most common causes of postpartum infection and neonatal sepsis. GBS causes both early and late-onset infections in newborns. Early GBS infections occur in the first week of life, while late disease occurs after the first week of life [13].

Some vaginal bacteria possess low virulence. They lead to chronic internal infections without obvious clinical signs. Once in the uterus, they cause the release of cytokines, prostaglandins, and metalloproteases. They can cause enlargement and shortening of the cervix, premature rupture of the amniotic sac (PRAS), increased uterine contractility and/or preterm birth (PB) [14].

Bacterial vaginosis (BV) implies colonization of the vagina with polymicrobial flora of complex communities that displace the normal flora, dominated by Lactobacillus spp. In bacterial vaginosis, there is no local inflammation, and no neutrophils are found in the vagina. In cases of BV, Gardnerella vaginalis, Mobiluncus spp., Anaerobic bacteria, Mycoplasma hominis, and Ureaplasma spp. are detected. The latter is the most common cause of vulvovaginitis in women 
of reproductive age and accounts for $45 \%$ of all vaginal infections. According to the US Public Health Service, the incidence of BV is 30\% in women of reproductive age [26]. The danger in this condition comes from the fact that these complex bacterial communities displace the normal flora, thus creating conditions for STIs and the development of other highly virulent microorganisms [15].

The purpose of the present study was to determine the bacterial profile of vaginal samples of pregnant women and the prevalence of the identified microorganisms.

\section{Materials and Methods}

A prospective epidemiological study (January 2019 - December 2020) was conducted on 150 pregnant women of Bulgarian origin, admitted to the Clinic of Obstetrics and Gynecology at the Dr. G. Stranski University Hospital -Pleven. The women were divided into two groups depending on the reason for hospitalization. The first group (group A) of pregnant women was admitted for treatment in the clinic $(n=83)$, in whom infectious genesis as a cause of complications could not be ruled out. The second group (group B) was admitted for childbirth $(n=63)$. Age distribution was as follows: women up to 20 years, from 21 to 35 years, and over 35 .

Vaginal secretion samples were taken from all the 150 women. Direct microscopy of a native preparation by wet mounting method to detect Trichomonas vaginalis and direct microscopy of a Gram-stained preparation was performed. We performed cultivation tests with an aerobic culture test (blood agar) to identify pathogenic bacteria and Sabouraud glucose agar (SGA) to detect the presence of Candida spp.

The vaginal samples were randomly collected from 107 women from both groups. Polymerase chain reaction (PCR) was performed, using a commercial kit based on the Taq-man principle: Real-time Quantitative PCR for DNATechnology for the following microorganisms: Mycoplasma genitalium, Mycoplasma hominis, Ureaplasma parvum, Ureaplasma urealyticum. Informed consent was obtained from all the women studied. Women with comorbidities (diabetes, autoimmune, thyroid disease, preeclampsia, eclampsia, and women with
IVF) were excluded from the study. The results were processed with a statistical office package STATGRAPHICS Plus and presented in tables and figures. Descriptive analysis was used to show the frequency distribution of the considered signs in tables, broken down by groups of research, graphic analysis - to visualize the results, and Fisher test and $\chi^{2}$ - test - to find the relationship between two categorical variables.

\section{Results}

The pregnant women included in the study, hospitalized in the Clinic of Obstetrics and Gynecology, were aged 15 to 41 (average $27 \pm$ sd 6.988). The pregnant women aged 21 to 35 years were the largest group -104 (69.33\%), and the smallest group was that of the women over 35 years: $12(10.67 \%)$. The percentage of pregnant women under 20 was significant - 34 women $(15.33 \%)$. The youngest one registered in this group was 15 .

Of the women hospitalized in the clinic, 42 (28\%) were uninsured, and those insured were significantly more - 108 women (72\%).

The clinical data of women showed that 83 (55.34\%) of them were admitted to the clinic for treatment, 63 (42\%) women - for childbirth without a history of complications related to the current pregnancy, and four women $(2.66 \%)$ were treated for complications of the puerperium. Of all the hospitalized and studied pregnant women, 87 (57.99\%) had medical problems during the current pregnancy and postpartum complications, and 63 women (42\%) gave birth without complications (Table 1).

The difference between the two studied groups of pregnant women A and B was statistically significant $(p<0.05)$. In this study, the women experiencing complications in the current pregnancies accounted for $57.99 \%$, against those without complications $(42.00 \%)$.

Table 2 presents the findings from the microbiological examination of hospitalized women.

Table 3 presents the distribution of Mycoplasmas in hospitalized pregnant women.

The total number and percentage exceed the total number of patients examined (107) because, in some of the patients, more than one causative agent was found. Representatives of Mycoplasma spp. (Figure 1). 
In the present study, the number of lactobacilli in the 150 pregnant women studied under a Gram microscope was normal in 43 women $(28.67 \%)$ and significantly lower in the remaining 107 women (71.34\%). Regarding epithelial cells, such were found in 39 women $(26.17 \%)$, while in $34(22.82 \%)$, none were detected. A problem arose in $75(50.34 \%)$ women with cytolysis data. We detected basal cells in one pregnant woman (0.67\%). Polymorphonuclear leukocytes (PMNL) - usually up to 5, were found in 63 women $(42.00 \%)$, moderately high (over 5 to $25)$ were found in 57 patients $(38.00 \%)$, and very high (over 25 PMNL) - in 30 women (20\%). Direct microscopy of Gram-stained preparation revealed bacteria in $86(57.32 \%)$ women. Of these, $24(16 \%)$ were Gram-negative rods, $28(18.66 \%)$ were Gram-positive cocci, and 34 (22.67\%) were Gram-negative and Gram variable polymorphic cocci. From the study of aerobic cultures (blood agar), microbiological growth was found in 49 patients. The established bacterial profile is presented in Figure 2.

E. coli was the most common microorganism we isolated and was found in $40.90 \%$ of the samples. Trichomonas vaginalis was detected in $20(13.33 \%)$ of the hospitalized women, and we saw single units of Candida albicans in 4 women $(2.67 \%)$, and the growth was moderate in 10 (6.67). The most significant findings were registered in 22 women (14.67\%). Out of a total of 150 women surveyed, $32(21.34 \%)$ had candidiasis.

We followed up the course of childbirth and the postpartum period in both groups for obstetric pathology during delivery (rupture of

Table 1. Distribution of pregnant women (n) by reasons for hospitalization

\begin{tabular}{lcc}
\hline Reasons for hospitalization & $\begin{array}{c}\text { Number of Women } \\
\text { (N) }\end{array}$ & $\begin{array}{c}\text { \%o of the total number of } \\
\text { hospitalizations }\end{array}$ \\
\hline For treatment & 83 & 55.34 \\
\hline For birth & 63 & 42 \\
\hline For treatment in the puerperium & 4 & 2.66 \\
\hline
\end{tabular}

Table 2. Vaginal bacterial profile in hospitalized pregnant women

\begin{tabular}{lcc}
\hline Bacterial finding & Group A & Group B \\
& n /\% & n /\% \\
\hline Reduced lactobacilli & $77 / 88.50$ & $30 / 47.61$ \\
\hline Epithelial cells with cytolysis & $75 / 86.20$ & $0 / 0.00$ \\
\hline High PMNL & $68 / 78.16$ & $19 / 30.15$ \\
\hline Presence of Bacteria & $62 / 71.26$ & $24 / 38.09$ \\
\hline Microbiological Growth & $43 / 40.95$ & $6 / 9.52$ \\
\hline Presence of Trichomonas vaginalis & $16 / 18.39$ & $4 / 6.34$ \\
\hline Presence of Candida & $21 / 24.13$ & $5 / 7.93$ \\
\hline
\end{tabular}

Table 3. Distribution of Mycoplasmas in hospitalized pregnant women

\begin{tabular}{lcc}
\hline $\begin{array}{l}\text { Mycoplasma spp. } \\
\text { Ureaplasma spp. }\end{array}$ & $\begin{array}{c}\text { Group A } \\
\mathbf{n} / \%\end{array}$ & $\begin{array}{c}\text { Group B } \\
\mathbf{n} / \mathbf{\%}\end{array}$ \\
\hline No Mycoplasma spp/Ureaplasma spp. & $42 / 48.27$ & $41 / 65.07$ \\
\hline Mycoplasma genitalium & $9 / 10.34$ & $4 / 6.34$ \\
\hline Ureaplasma parvum & $39 / 44.28$ & $18 / 28.67$ \\
\hline Ureaplasma urealyticum & $19 / 21.83$ & $9 / 14.28$ \\
\hline Mycoplasma hominis & $23 / 26.43$ & $10 / 15.87$ \\
\hline
\end{tabular}




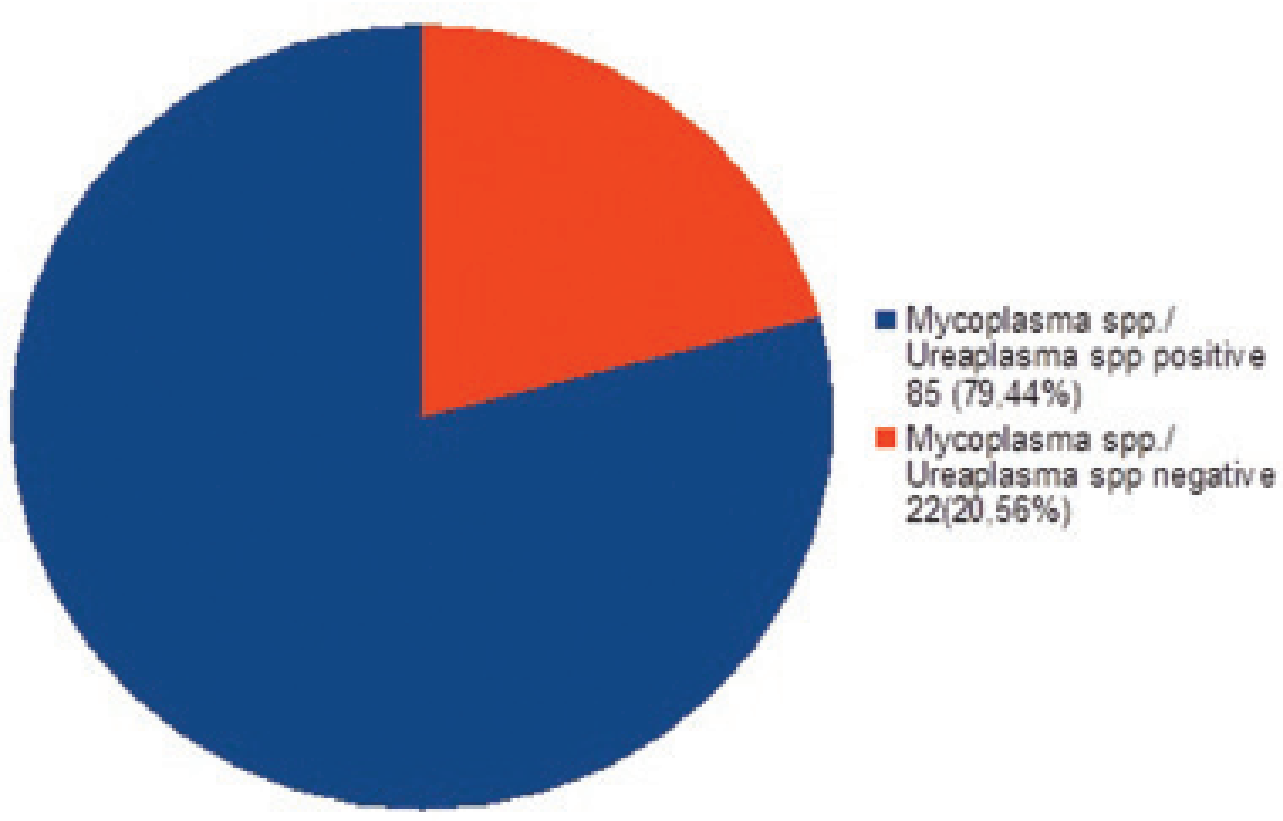

Figure 1. Mycoplasma spp./Ureaplasma spp. in women with established bacterial vaginosis

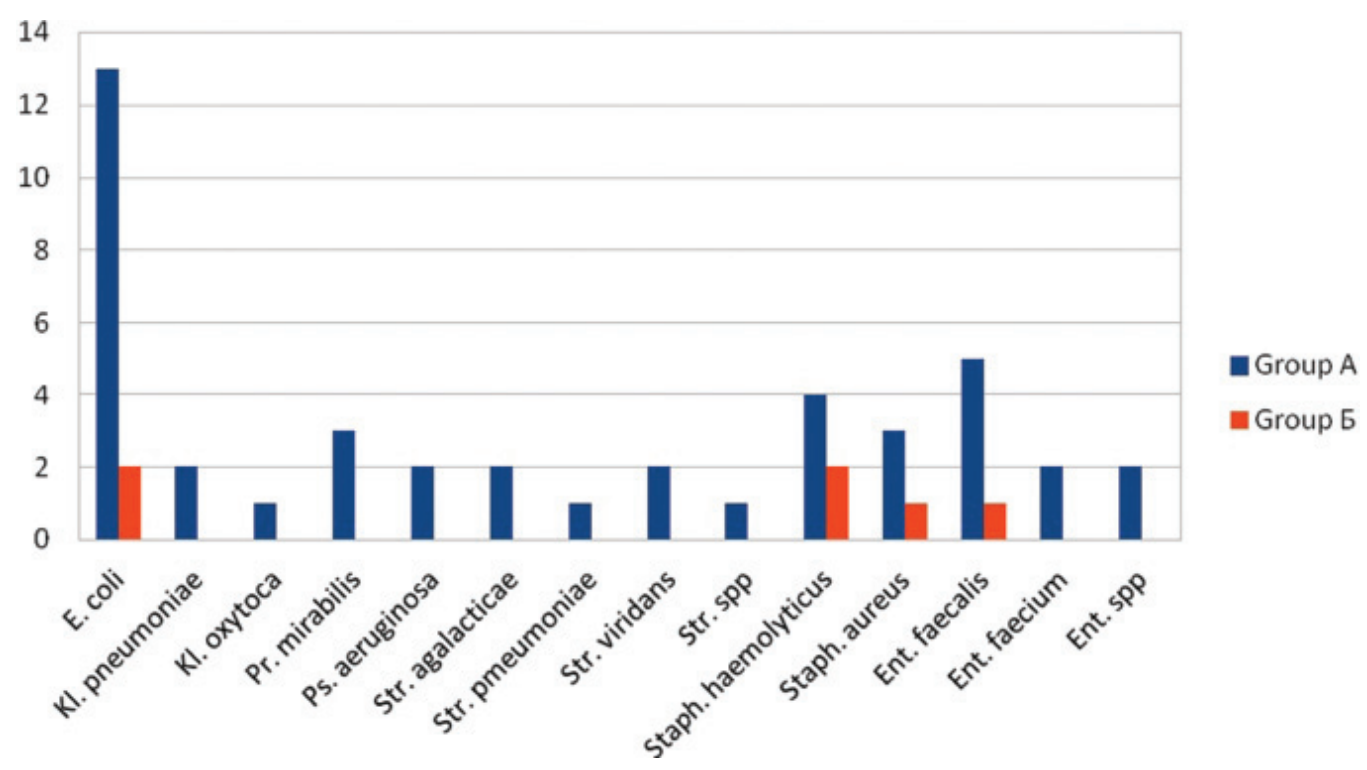

Figure 2. Bacterial profile in pregnant women

soft tissues, violation of uterine contractility, retained placental parts), and pathology during the puerperium (fever, delayed involution, endometritis). In group $\mathrm{A}$, complications of childbirth occurred in 33 women $(31.42 \%)$, and $72(68.57 \%)$ had no problems, respectively (P$\left.0.3153, \chi^{2}=7.06\right)$. No statistically significant difference was found. Of the women from group B, 11 women $(24.44 \%)$ had complications of childbirth, no complications occurred in
$33(73.33 \%)$, and we found no statistically significant difference $\left(\mathrm{P}-0.3153, \chi^{2}=7.06\right)$. In the women of group A, those with complications in the puerperium were $63(60 \%)$, and $42(40 \%)$ had no complications, respectively $\left(\mathrm{P}-0.1563, \chi^{2}\right.$ 7.92). In group $\mathrm{B}$, the women with complications in the puerperium were $26(57.78 \%)$, and those without were $19(42.22 \%)$, respectively. No statistically significant difference (P-0.7265 $\chi^{2}$ 3.63) was found. 


\section{Discussion}

Despite the general trend of declining birth rates among young $(<20$ years) girls, teenage pregnancy continues to be a significant public health problem worldwide. The period between 15 and 35 years is considered the fertile age in our country and worldwide. According to many authors, adulthood begins after 20. In the United States, births to young (15-19 years) and older ( $\geq 35$ years) women were $12.6 \%$ and $11.4 \%$, respectively, and an increased risk of fetal death after 24 weeks. Abortions, stillbirths, premature births, and low birth weight are among the most commonly cited complications in pregnant teenagers. Pregnant women over the age of 35 are at an increased risk of accompanying hypertension and renal and endocrine diseases. Many of them become pregnant after sterility or infertility. This group is characterized by an increased frequency of genetic and chromosomal abnormalities. The problem with uninsured patients in our country is the limited access to medical care and monitoring during pregnancy.

The difference between the two studied groups of pregnant and hospitalized women was statistically significant $(p<0.05)$. In the current study, women with a complicated pregnancy course prevailed and accounted for $57.99 \%$ vs. $42 \%$.

Hyman et al. (2014) showed that ethnicity might be an essential factor in determining specific aspects of vaginal microbial composition during pregnancy [16]. The postpartum period is associated with a decrease in Lactobacillus spp., especially L. Crispatus, and an increase in the number of species associated with bacterial vaginosis (BV), including Prevotella spp., Finegodia magna, Streptococcus anginosus, and other rarer species $[17,18]$.

It is known that Lactobacillus spp. plays a key protective role by producing various bacteriostatic and bactericidal compounds or competitively excluding other bacterial species. The predominant proportion of these bacteria is up to $95 \%$. The following species are favoured during pregnancy: Lactobacillus iners, Lactobacillus crispatus, Lactobacillus jensenii, and Lactobacillus johnsonii. A significant reduction in lactobacilli below five or no lactobacilli increases the risk of vaginal infections and premature birth. Our data showed that a substantial proportion of the women surveyed were at such a risk. The studies of MacIntyre D. et al. on pregnant and postpartum women six weeks after birth have shown that the vaginal microbiome composition changes dramatically during pregnancy and after birth. It becomes less dominant than Lactobacillus spp. with increased alpha diversity, regardless of community structure during pregnancy and ethnicity [8].

In our study, a significant proportion of the women surveyed were exposed to such a risk. The reduced amounts of lactobacilli we found $(88.5 \%)$ and the large number of cases $(86.2 \%)$ with cytolysis and high levels of PMNL in $78.10 \%$ proved the presence of inflammatory processes in women in group A. This finding correlates with the high rate of complications during pregnancy $(31.42 \%)$ and the puerperium $(60 \%)$.

E. coli is a common cause of sepsis during pregnancy [19]. The incidence of E. coli infection is estimated between 0.2 and 5 per 1000 live births [20]. Newborn colonization is often the result of maternal transmission during childbirth [21]. Our results showed that the highest percentage of E. coli was in group A (30.61\%), and the high levels indicated a real risk of complications in both pregnant and postpartum women and newborns.

Enterococcus spp. and E. faecalis, in particular, are among the most common causes of endocarditis, postpartum endometritis, and abortion [22]. The risk of colonization with multidrug-resistant enterococci is increased in premature infants [23]. In premature infants, enterococcal infections can be life-threatening [24,25]. The colonization of the newborn with microorganisms begins during birth. The first colonizing microorganisms originate from the vaginal and gastrointestinal flora of the mother, ingested through the mouth $[23,26]$. Our study also found frequent carriers ( 9 cases in group A and 1 in group B). In a survey by Ballén V. et al., the prevalence of $\mathrm{K}$. pneumoniae in pregnant women was $0.6 \%$ [27]. Many authors have reported an increase in virulent strains of $\mathrm{K}$. pneumoniae leading to acute placental infection, chorioamnionitis, and intrauterine fetal death $[28,29]$. Several reports have suggested that 
Haemophilus influenzae can cause serious illness in pregnant women, often leading to fetal death or premature birth with severe disease in the newborn [30,31]. Our study also identified 3 cases of Klebsiella spp. Puerperal sepsis caused by group A streptococci (GAS) remains a significant cause of maternal and infant mortality worldwide. They can lead to sepsis and are associated with a very high mortality rate (30$50 \%$ ) [32]. Streptococci were detected in 6 of the pregnant women we studied. According to the literature, trichomoniasis infection in pregnant women varies from 5 to $15 \%[33,34]$. The frequency of $13 \%$ we established does not exceed the frequency cited in other publications. Data from other studies on C. albicans have shown that the prevalence of this infection among pregnant women varies from 20 to $25 \%$ [35]. Our data established a frequency of $24.13 \%$ among the hospitalized pregnant women of group A.

All disorders in the vaginal flora with excessive bacterial growth, epithelial cells with cytolysis, high PMNL, Gram (-) and Gram variable bacteria, and reduction or absence of lactobacilli are associated with high $\mathrm{pH}$. Many authors describe them as bacterial vaginosis. A positive correlation between bacterial vaginosis and preterm birth has been established in a prospective study of singleton pregnancies after the 20th gestation week. The incidence of spontaneous previous births $(<37$ weeks) among pregnant women with bacterial vaginosis (BV) was 3.8\% [36]. It is the most common cause of vulvovaginitis in women of reproductive age, accounting for $45 \%$ of all vaginal infections. According to the US Public Health Service, the incidence of BV is 30\% in women of reproductive age [37]. There is considerable evidence that bacterial vaginosis during pregnancy is associated with poor perinatal outcomes. During pregnancy, bacterial vaginosis increases the risk of sepsis, early miscarriage, normal abortion, late miscarriage, premature birth, PPOM, and histological chorioamnionitis. The presence of Mycoplasma spp./Ureaplasma spp. probably has a direct effect on the development of bacterial vaginosis. Our study found $80 \%$ carriership of the four species Mycoplasma spp./Ureaplasma spp. In some of the women, more than one causative agent was identified. We found relatively similar results regarding complications during pregnancy (31.42\%) for group A and (24.44\%) for group $\mathrm{B}$, so we cannot exclude infectious agents as the cause for these results. Regarding postpartum complications, the frequency was also similar: $60 \%$ vs. $57.78 \%$. These complications may also be associated with infectious genesis.

\section{Conclusions}

Bacterial vaginal colonization is a major medical and social problem. Our study on the profile of the vaginal microbiota in pregnant women and the spread of pathogenic microorganisms confirms that the high rate of colonization with microorganisms poses a risk for developing a maternal-fetal infection.

\section{Acknowledgments}

The study was conducted under a research project № 5/2020, funded by Medical University Pleven, Bulgaria.

\section{References}

1. Ursell LK, Metcalf JL, Parfrey LW, Knight R. Defining the human microbiome. Nutr Rev. 2012;70 Suppl 1(Suppl 1):S38-S44.

2. Mendling W. Vaginal Microbiota. Adv Exp Med Biol. 2016;902:83-93.

3. Vicariotto F, Mogna L, Del Piano $M$. Effectiveness of the two microorganisms Lactobacillus fermentum LF15 and Lactobacillus plantarum LP01, formulated in slow-release vaginal tablets, in women affected by bacterial vaginosis: a pilot study. J Clin Gastroenterol. 2014 Nov-Dec;48 Suppl 1:S10612.

4. Smith SB, Ravel J. The vaginal microbiota, host defence and reproductive physiology. J Physiol. 2017;595(2):451-63.

5. Goldenberg RL, McClure EM, Saleem S, Reddy UM. Infection-related stillbirths. Lancet (London, England). 2010 Apr;375(9724):148290.

6. Loiwal V, Kumar A, Gupta P, Gomber S, Ramachandran VG. Enterobacter aerogenes outbreak in a neonatal intensive care unit. Pediatr Int. 1999 Apr;41(2):157-61.

7. Dalben M, Varkulja G, Basso M, Krebs VL, Gibelli MA, van der Heijden I, et al. 
Investigation of an outbreak of Enterobacter cloacae in a neonatal unit and review of the literature. J Hosp Infect. 2008 Sep;70(1):7-14.

8. Akbari M, Bakhshi B, Najar Peerayeh S. Particular Distribution of Enterobacter cloacae Strains Isolated from Urinary Tract Infection within Clonal Complexes. Iran Biomed J. 2016;20(1):49-55.

9. Ghasemi, E., Mansouri, S., Shahabinejad, N. Vaginal Colonization and Susceptibility to Antibiotics of Enterococci During Late Pregnancy in Kerman City, Iran. Arch. Clin. Infect. 2016 Oct; 11(4).

10. Ghotaslou R, Ghorashi Z, Nahaei MR. Klebsiella pneumoniae in neonatal sepsis: a 3-year-study in the pediatric hospital of Tabriz, Iran. Jpn J Infect Dis. 2007 May;60(2-3):126-8. PMID: 17515647.

11. Perdue DG, Bulkow LR, Gellin BG, Davidson M, Petersen KM, Singleton RJ, et al. Invasive Haemophilus influenzae disease in Alaskan residents aged 10 years and older before and after infant vaccination programs. JAMA. 2000 Jun 21;283(23):3089-94.

12. Berndsen MR, Erlendsdóttir H, Gottfredsson M. Evolving epidemiology of invasive Haemophilus infections in the post-vaccination era: results from a long-term population-based study. Clin Microbiol Infect. 2012 Sep;18(9):918-23.

13. Phares, C.R., Lynfield, R., Farley, M.M., et al. Active Bacterial Core surveillance/Emerging Infections Program Network. Epidemiology of invasive group B streptococcal disease in the United States. 1999-2005. JAMA. 2008 May 7;299(17):2056-65.

14. Laxmi U, Agrawal S, Raghunandan C, Randhawa VS, Saili A. Association of bacterial vaginosis with adverse fetomaternal outcome in women with spontaneous preterm labor: a prospective cohort study. J Matern Fetal Neonatal Med. 2012 Jan;25(1):64-7.

15. Krauss-Silva L, Almada-Horta A, Alves MB, Camacho KG, Moreira ME, Braga A. Basic vaginal $\mathrm{pH}$, bacterial vaginosis and aerobic vaginitis: prevalence in early pregnancy and risk of spontaneous preterm delivery, a prospective study in a low socioeconomic and multiethnic South American population. BMC Pregnancy Childbirth. 2014 Mar 19;14:107.

16. Hyman, R. W., Fukushima M., Jiang H., et al. Diversity of the vaginal microbiome correlates with preterm birth. Reprod Sci. 2014;21(1):3240.

17. Aagaard K, Riehle K, Ma J, Segata N, Mistretta TA, Coarfa C, et al. A metagenomic approach to characterization of the vaginal microbiome signature in pregnancy. PLoS One. 2012;7(6):e36466.

18. MacIntyre DA, Chandiramani M, Lee YS, Kindinger L, Smith A, Angelopoulos N, et al. The vaginal microbiome during pregnancy and the postpartum period in a European population. Sci Rep. 2015;5(1):1-9.

19. Knowles SJ, O'Sullivan NP, Meenan AM, Hanniffy R, Robson M. Maternal sepsis incidence, aetiology and outcome for mother and fetus: a prospective study. BJOG. 2015 Apr;122(5):663-71.

20. Bradley, JS. Neonatal infections. Pediatr Infect Dis. 1985 May-Jun;4(3):315-20.

21. McCracken GH Jr, Sarff LD, Glode MP, Mize SG, Schiffer MS, Robbins JB, et al. Relation between Escherichia coli K1 capsular polysaccharide antigen and clinical outcome in neonatal meningitis. Lancet. 1974 Aug 3;2(7875):246-50.

22. Tharpe N. Postpregnancy genital tract and wound infections. J Midwifery Womens Health. 2008 May-Jun;53(3):236-46.

23. Mackie RI, Sghir A, Gaskins HR. Developmental microbial ecology of the neonatal gastrointestinal tract. Am J Clin Nutr. 1999 May;69(5):1035S-1045S.

24. Murray BE. Vancomycin-resistant enterococcal infections. N Engl J Med. 2000 Mar 9;342(10):710-21.

25. Maki DG, Agger WA. Enterococcal bacteremia: clinical features, the risk of endocarditis, and management. Medicine (Baltimore). 1988 Jul;67(4):248-69.

26. Orrhage K, Nord CE. Factors controlling the bacterial colonization of the intestine in breastfed infants. Acta Paediatr Suppl. 1999 Aug;88(430):47-57.

27. Ballén V, Sáez E, Benmessaoud R, Houssain T, Alami H, Barkat A, et al. First report of a Klebsiella pneumoniae ST466 strain causing neonatal sepsis harbouring the blaCTX-M-15 gene in Rabat, Morocco. FEMS Microbiol Lett. 2015 Jan;362(1):1-4.

28. Torabi R, Charnova S, Abellar RG, Pinar H, De Paepe ME. Intrauterine infection with Klebsiella pneumoniae: report of a case and literature review. Pediatr Dev Pathol. 2008 MarApr;11(2):152-5.

29. Sheikh SS, Amr SS, Lage JM. Acute placental infection due to Klebsiella pneumoniae: report of a unique case. Infect Dis Obstet Gynecol. 2005 Mar;13(1):49-52.

30. Wallace RJ Jr, Baker CJ, Quinones FJ, Hollis DG, Weaver RE, Wiss K. Nontypable Haemophilus influenzae (biotype 4) as a neonatal, maternal, and genital pathogen. Rev Infect Dis. 1983 JanFeb;5(1):123-36. 
31. Warren S, Tristram S, Bradbury RS. Maternal and neonatal sepsis caused by Haemophilus influenzae type d. J Med Microbiol. 2010 Mar;59(Pt 3):370-2.

32. Sitkiewicz, I., Green, N.M., Guo, N. et al. Adaptation of group A Streptococcus to human amniotic fluid. PLoS One. 2010 Mar 23;5(3):e9785.

33. Coleman JS, Gaydos CA, Witter F. Trichomonas vaginalis vaginitis in obstetrics and gynecology practice: new concepts and controversies. Obstet Gynecol Surv. 2013 Jan;68(1):43-50.

34. M Domeika, L Zhurauskaya, Savicheva, N Frigo, E Sokolovskiy, A Hallén, et al. Guidelines for the laboratory diagnosis of trichomoniasis in East European countries. JEADV. 2010 October;24(10):1125-34.

35. Foxman B, Muraglia R, Dietz JP, Sobel JD, Wagner J. Prevalence of recurrent vulvovaginal candidiasis in 5 European countries and the United States: results from an internet panel survey. J Low Genit Tract Dis. 2013 Jul;17(3):340-5.
36. Krauss-Silva L, Almada-Horta A, Alves MB, Camacho KG, Moreira ME, Braga A. Basic vaginal $\mathrm{pH}$, bacterial vaginosis and aerobic vaginitis: prevalence in early pregnancy and risk of spontaneous preterm delivery, a prospective study in a low socioeconomic and multiethnic South American population. BMC Pregnancy Childbirth. 2014 Mar 19;14:107.

37. Keskinoglu P, Bilgic N, Picakciefe M, Giray H, Karakus N, Gunay T. Perinatal outcomes and risk factors of Turkish adolescent mothers. J Pediatr Adolesc Gynecol. 2007 Feb;20(1):1924. 